\title{
Monoclonal gammopathy of renal significance (MGRS)-related AL amyloidosis complicated by amyloid myopathy: a case report
}

Erina Ono ${ }^{1}$, Akira Ishii ${ }^{1 *}$, Yoshiaki Higashi ${ }^{1}$, Natsuko Koita ${ }^{2}$, Takashi Ayaki ${ }^{2}$, Katsuya Tanigaki ${ }^{1}$, Shunsuke Takayanagi ${ }^{1}$, Naoya Kondo ${ }^{1}$, Kaoru Sakai ${ }^{1}$, Shuichiro Endo ${ }^{1}$, Hideki Yokoi ${ }^{1}$, Takeshi Matsubara', Sachiko Minamiguchi ${ }^{3}$, Ichizo Nishino ${ }^{4}$, Ryosuke Takahashi ${ }^{2}$ and Motoko Yanagita'

\begin{abstract}
Background: Lately, monoclonal gammopathy of renal significance (MGRS) has been defined as a group of renal disorders that are strongly associated with monoclonal protein, including amyloid immunoglobulin light chain (AL) amyloidosis. Amyloid myopathy is rare (1.5\% of all patients with amyloidosis) and the prognosis is poor.

Furthermore, only approximately $20 \%$ of patients with amyloid myopathy are reported to have renal involvement, indicating a lack of data in the literature.

Case presentation: Here, we report a rare case of MGRS-related AL amyloidosis complicated by amyloid myopathy that presented with muscle weakness in the upper and lower limbs, neck and fingers, and nephrotic syndrome. Blood, urine, and bone marrow examination revealed monoclonal gammopathy of undetermined significance (MGUS) (Bence Jones protein-lambda). Muscle biopsy of the vastus lateralis muscle demonstrated amyloid proteins in the sarcolemma and in the blood vessel walls on Congo red staining, suggesting amyloid myopathy, and tiny inclusions in fibers on modified Gomori trichrome stain. Although we thought they were reminiscent of nemaline bodies, we could not confirm the nature of this structure. Renal biopsy demonstrated amyloid proteins in the mesangial region, part of the capillary walls, and the blood vessel walls on direct fast scarlet staining. As these amyloid proteins were positive for p-component staining and negative for amyloid A staining, $\beta 2$-microglobulin, and pre-albumin, and as lambda light chains were positive in the mesangial region, we diagnosed the patient with MGRS-related AL amyloidosis. Although he was treated with melphalan and dexamethasone, his symptoms did not improve.
\end{abstract}

Conclusions: AL amyloidosis involving the kidneys and muscles has a poor prognosis, and a delayed diagnosis of amyloid myopathy is common because of its rarity and frequent misdiagnosis, which increases organ function deterioration. Therefore, early detection, therapeutic intervention, and careful follow-up are crucial.

Keywords: Amyloidosis, Amyloid myopathy, Monoclonal gammopathy of undetermined significance, Monoclonal gammopathy of renal significance, Sporadic late-onset nemaline myopathy

\footnotetext{
* Correspondence: aish@kuhp.kyoto-u.ac.jp

'Department of Nephrology, Graduate School of Medicine, Kyoto University,

54 Shogoin Kawahara-cho, Sakyo-ku, Kyoto 606-8507, Japan

Full list of author information is available at the end of the article
}

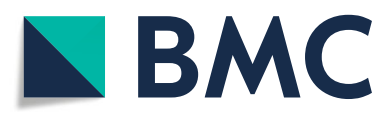

(c) The Author(s). 2021 Open Access This article is licensed under a Creative Commons Attribution 4.0 International License, which permits use, sharing, adaptation, distribution and reproduction in any medium or format, as long as you give appropriate credit to the original author(s) and the source, provide a link to the Creative Commons licence, and indicate if changes were made. The images or other third party material in this article are included in the article's Creative Commons licence, unless indicated otherwise in a credit line to the material. If material is not included in the article's Creative Commons licence and your intended use is not permitted by statutory regulation or exceeds the permitted use, you will need to obtain permission directly from the copyright holder. To view a copy of this licence, visit http://creativecommons.org/licenses/by/4.0/. The Creative Commons Public Domain Dedication waiver (http://creativecommons.org/publicdomain/zero/1.0/) applies to the data made available in this article, unless otherwise stated in a credit line to the data. 


\section{Background}

Monoclonal gammopathy of renal significance (MGRS) has been defined in recent years as a group of renal disorders that are strongly associated with monoclonal protein ( $M$ protein), including amyloid immunoglobulin light chain (AL) amyloidosis, light-chain deposition disease, immunotactoid glomerulonephritis, and proliferative glomerulonephritis with monoclonal Ig deposits (PGNMID) [1-3]. The concept of MGRS is attracting attention because the significance of early treatment for MGRS-related kidney disease targeting the responsible small B-cell clones has been reported in terms of preventing renal deterioration [4]. AL amyloidosis is characterized by the presence of monoclonal protein and amyloid deposits in various organs $[5,6]$. Although involvement of the kidney, heart, liver, and nervous system commonly occurs in systemic amyloidosis [5], it has been reported that the complication of amyloid myopathy is rare $(1.5 \%)$ and the prognosis is poor (approximately 22-32 months from onset of symptoms to death) [7-9]. Furthermore, only 16 to $25 \%$ of patients with amyloid myopathy are reported to have renal involvement $[7,10]$. There is a scarcity of data on amyloid myopathy with nephropathy in the scientific literature. Nemaline myopathy is a group of muscle diseases pathologically characterized by the presence of nemaline rods in myofibers on modified Gomori trichrome stain. Sporadic late-onset nemaline myopathy (SLONM) affects adults in the middle age to elderly period and involves subacutely progressive weakness and atrophy in the axial and predominantly proximal limb muscles [11]. Approximately half of SLONM patients have concomitant monoclonal gammopathy of undetermined significance (MGUS), and the prognosis of SLONM with MGUS (SLONM-MGUS) is reported to be poor mainly due to respiratory failure, leading to death usually within a few years [12].

Here, we report a rare case of MGRS-related AL amyloidosis complicated by amyloid myopathy detected by upper and lower limb, neck flexion and extension, flexor and extensor digitorum muscle weakness, and nephrotic syndrome.

\section{Case presentation}

An 83-year-old man had a two-year history of proteinuria $(2+)$ that had become worse in the year before admission. He complained of progressive proximal lower limb muscle weakness for 10 months before admission. He was admitted to our hospital because of upper and lower limb, neck flexor and extensor, and flexor and extensor digitorum muscle weakness, and pitting edema of the legs. He had a medical history of atrial fibrillation and hypertension. He had smoked five cigarettes per day for the decade before he turned 30 .
On admission, predominantly proximal symmetrical muscle weakness and leg pitting edema were observed. His manual muscle testing (MMT) scores were: neck flexion $=4$; neck extension $=4$; deltoids $=3$; biceps brachii $=3$; triceps brachii $=3$; flexor and extensor muscles of the hand $=4$; flexor and extensor digitorum muscle $=$ 3 ; iliopsoas $=2$; gluteus maximus $=2$; quadriceps $=2$; hamstring $=2$; flexor and extensor muscles of the foot $=$ 5. His blood pressure was $120 / 80 \mathrm{mmHg}$. Laboratory tests on admission showed serum creatinine and albumin levels of $0.7 \mathrm{mg} / \mathrm{dL}$ and $2.9 \mathrm{~g} / \mathrm{dL}$, respectively, and his urinary protein creatinine ratio was $7.3 \mathrm{~g} / \mathrm{gCr}$, suggesting nephrotic syndrome. Serum creatine kinase $(820$ $\mathrm{U} / \mathrm{L}$ ), aldolase (13.7 IU/L), and urinary myoglobin levels were initially elevated, but after admission they normalized and myoglobinuria disappeared. All autoantibodies (i.e., antinuclear antibodies [ANA], anti-double stranded DNA [anti-dsDNA] antibodies, anti-Ro/SS-A antibodies, anti-La/SS-B antibodies, anti-aminoacyl tRNA synthetase [anti-ARS] antibodies, anti-cyclic citrullinated peptide [anti-CCP] antibodies, anti-mitochondrial M2 [AMA2] antibodies, acetylcholine receptor $[\mathrm{AChR}]$ antibodies, and anti-muscle-specific kinase [anti-MuSK] antibodies) were negative. Although serum immunoglobulin (Ig) G was decreased $(705 \mathrm{mg} / \mathrm{dL})$, IgA $(159 \mathrm{mg} / \mathrm{dL})$ and $\operatorname{IgM}$ $(37 \mathrm{mg} / \mathrm{dL})$ were within normal range. Bence Jones protein (BJP)-lambda was observed in both the blood and urine, the serum free light chain lambda was $533 \mathrm{mg} / \mathrm{L}$, the serum kappa/lambda ratio was 0.046 , and the $M$ protein level was $0.575 \mathrm{~g} / \mathrm{dL}$. The plasma cell count in the bone marrow was $0.7 \%$, indicating MGUS. Magnetic resonance imaging (MRI) scan showed proximal lower limb muscle atrophy and edema, including the quadriceps femoris, obturator, and gluteus maximus muscles (Fig. 1). Needle electromyogram was performed in the first dorsal interosseous, deltoid, and iliopsoas muscles. Although it showed fibrillation potential and positive sharp waves, it also showed complex repetitive discharge, low amplitude, and early recruitment, suggestive of myogenic changes. Nerve conduction study of the median, ulnar, tibial, and sural nerves was normal (Table 1). Therefore, the patient was diagnosed with myopathy as the cause of the predominantly proximal symmetrical muscle weakness.

A muscle biopsy of the right vastus lateralis muscle was performed on the 25th day of admission. Light microscopy showed a small cluster of atrophic angulated fibers in addition to mild fiber size variation on hematoxylin and eosin (HE)-stained sections. On nicotinamide adenine dinucleotide-tetrazolium reductase (NADH-TR), some fibers minimally lacked oxidative activity (Fig. 2a). On modified Gomori trichrome, one atrophic fiber was filled with fine cytoplasmic inclusions (Fig. 2b). Although we thought they were reminiscent of 

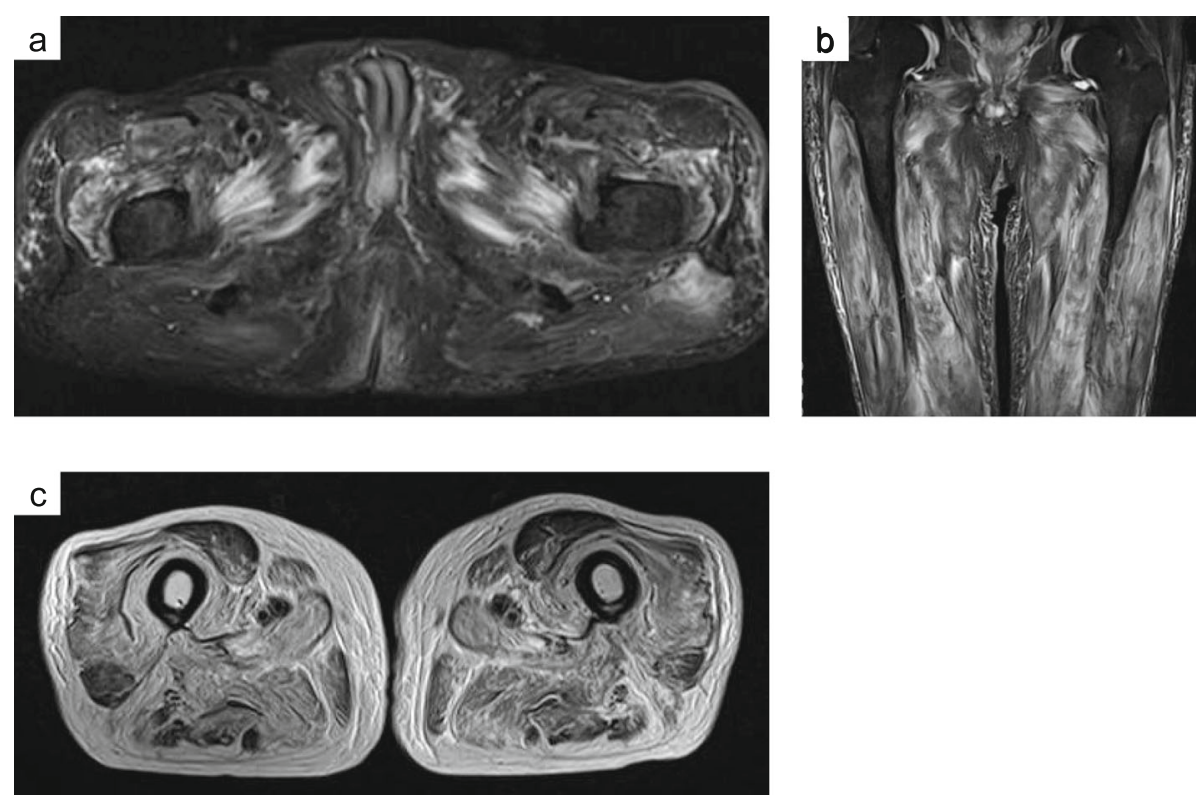

Fig. 1 Muscle magnetic resonance imaging scan images. Axial (a) and coronal (b) short inversion time inversion recovery (STIR) images and axial T2 weighted image (c) show muscle atrophy and high intensity signals in the quadriceps femoris, obturator, and gluteus maximus muscles

nemaline bodies, we could not confirm the nature of this structure as a sample for electron microscopic study was not available. Congo red positivity in the sarcolemma membrane and vascular walls was observed (Fig. 2c, d, e). Immunofluorescence staining of frozen muscle tissue showed that lambda and kappa light chains were negative (Fig. 3). Considering the clinical course and histopathological findings, the patient was diagnosed with amyloid myopathy.

A percutaneous renal biopsy was performed on the 35th day of admission to determine the cause of nephrotic syndrome. Light microscopy showed an accumulation of eosinophilic, amorphous deposits in the mesangial region and part of the capillary walls in HEstained sections (Fig. 4a), periodic acid-Schiff-stained sections (Fig. 4b), and periodic acid-methenamine-silver-stained sections (Fig. 4c), which were determined to be amyloid proteins as they were stained positive by direct fast scarlet staining (Fig. 4d). Amyloid proteins were also observed in the blood vessel walls. As these amyloid proteins were positive for $\mathrm{p}$-component staining and negative for amyloid A staining, $\beta 2$-microglobulin, and

Table 1 Nerve conduction study

\begin{tabular}{|c|c|c|c|c|}
\hline Motor nerves/site & Amplitude (mV) & Latency (ms) & $\mathrm{NCV}(\mathrm{m} / \mathrm{s})$ & F-wave minimal latency (ms) \\
\hline \multicolumn{5}{|l|}{ Median nerve } \\
\hline Wrist & 5.7 & 3.6 & & 25.1 \\
\hline Elbow & 5.4 & 7.2 & 54.9 & \\
\hline \multicolumn{5}{|l|}{ Ulnar nerve } \\
\hline Wrist & 6.1 & 2.8 & & 24.8 \\
\hline Below elbow & 5.9 & 5.8 & 65.1 & \\
\hline Above elbow & 5.7 & 7.1 & 56 & \\
\hline \multicolumn{5}{|l|}{ Tibial nerve } \\
\hline Ankle & 4.6 & 3.8 & 44.9 & 44.8 \\
\hline Popliteal fossa & 3.3 & 11.8 & & \\
\hline Sensory nerves & Amplitude $(\mu \mathrm{V})$ & Latency (ms) & $\mathrm{NCV}(\mathrm{m} / \mathrm{s})$ & \\
\hline Median nerve & 24.4 & 2.8 & 57 & \\
\hline Ulnar nerve & 16.1 & 2.3 & 60 & \\
\hline Sural nerve & \multicolumn{4}{|c|}{ Not evoked (*due to leg edema) } \\
\hline
\end{tabular}



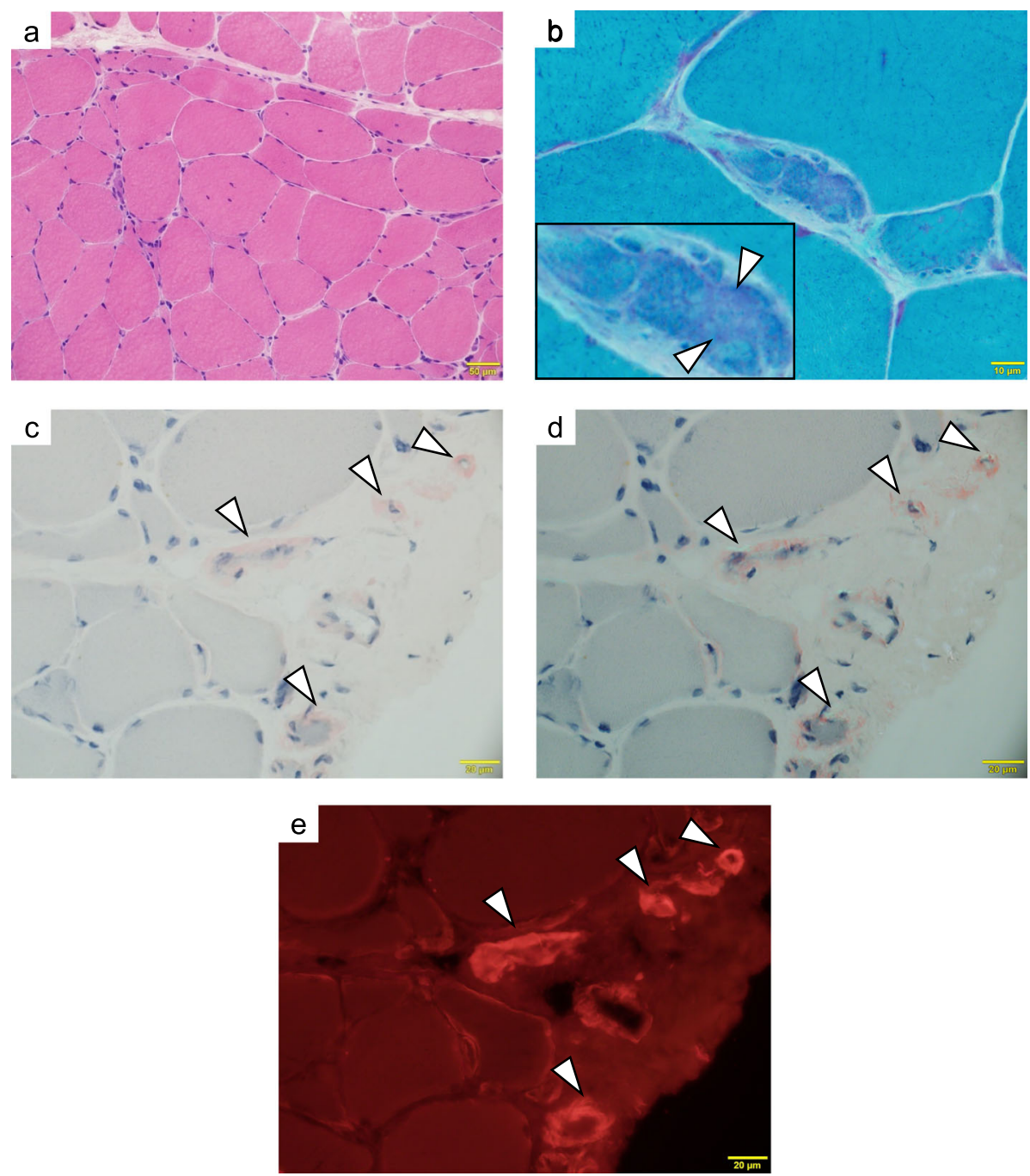

Fig. 2 Muscle biopsy specimen on light microscopy. Hematoxylin and eosin staining (a) shows a small cluster of atrophic angulated fibers and mild fiber size variation. Modified Gomori trichrome stain (b) shows tiny inclusions in fibers (white arrowheads). Congo red staining (c) shows amyloid proteins in which birefringence is observed under the polarizing microscope (d) and which is positive under the fluorescence microscope (e) in the sarcolemma and blood vessel walls (white arrowheads)

pre-albumin, we diagnosed the patient with AL amyloidosis. Approximately $40 \%$ interstitial fibrosis and tubular atrophy was observed. Immunofluorescence staining of frozen tissue showed that lambda light chains were positive in the mesangial region (Fig. 5a). Staining with IgG, IgA, IgM, complement component 3 (C3)c, C1q, and kappa light chains were negative (Fig. 5b). Electron microscopy showed amyloid fibrils in the mesangial region (Fig. 6). Thus, the patient was diagnosed with AL amyloidosis caused by BJP-lambda.

Each examination was subsequently performed for systemic amyloidosis. Although echocardiography showed mild left ventricular diastolic dysfunction without characteristic findings for cardiac amyloidosis such as a classical granular sparkling appearance, we did not exclude cardiac amyloidosis because 1) both serum NT-pro brain natriuretic peptide $(\mathrm{BNP})(369.7 \mathrm{pg} / \mathrm{mL})$ and troponin $\mathrm{T}$ levels $(0.166 \mathrm{ng} / \mathrm{mL})$ were elevated and 2) the patient had a history of atrial fibrillation. Amyloid protein was negative in the colon biopsy. Finally, the patient was diagnosed with AL amyloidosis involving the kidneys, muscles, and possibly the heart.

The patient was treated with MD (melphalan, $8 \mathrm{mg}$ and dexamethasone, $20 \mathrm{mg}$ daily for 4 days) therapy for AL amyloidosis. Although his cardiac function and respiratory function did not decline, the muscle weakness grew worse, and the nephrotic syndrome, renal insufficiency, and serum free light chain level did not improve. Twenty-one months after therapy commenced, the serum creatinine level had increased to $1.2 \mathrm{mg} / \mathrm{dL}$, and his MMT scores were: neck flexion $=3$; neck extension $=$ 3 ; deltoids $=2-3$; biceps brachii $=2-3$; triceps brachii $=2-$ 

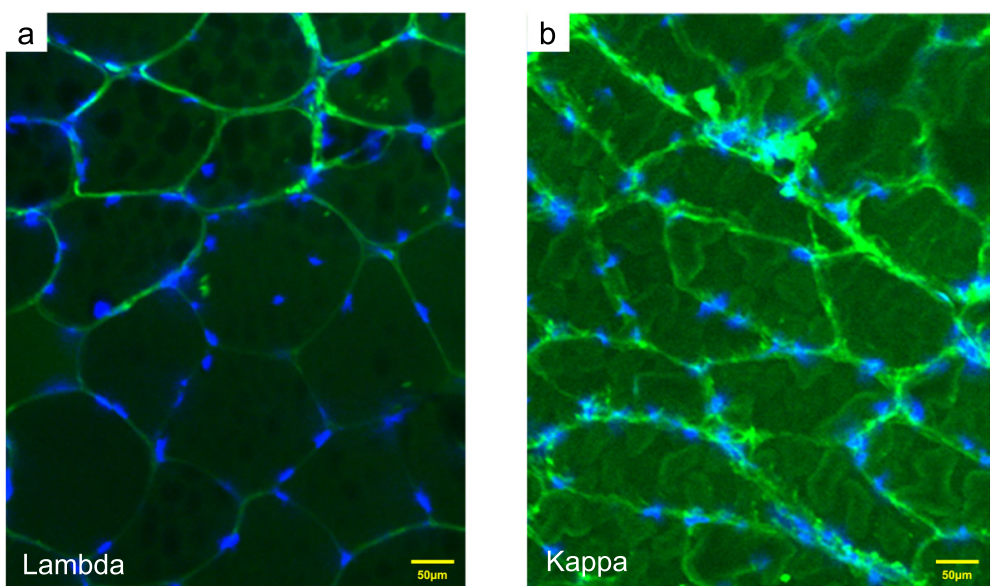

Fig. 3 Muscle biopsy specimen on immunofluorescence microscopy. Blue indicates 4',6-diamidino-2-phenylindole and green indicates lambda (a) and kappa (b), both of which are negative

3; flexor and extensor muscles of the hand $=2-3$; flexor and extensor digitorum muscle $=2-3$; iliopsoas $=1-2$; gluteus maximus $=1-2$; quadriceps $=1-2$; hamstring $=1-2$; flexor and extensor muscles of the foot $=2-3$.

\section{Discussion and conclusions}

Here, we report a rare case of drug-resistant MGRSrelated AL amyloidosis complicated by amyloid myopathy.

We could identify the cause of the muscle weakness and nephrotic syndrome through muscle and renal biopsies. The complication of amyloid myopathy is rare. In previous reports, only $1.5 \%$ of $\mathrm{AL}$ amyloidosis patients had amyloid myopathy, and only 16 to $25 \%$ of those with amyloid myopathy had renal involvement [7, 10]; the percentage of men was $71 \%$ and the median age was 67 years [7]. Common symptoms of amyloid myopathy include predominantly proximal muscle weakness, muscle pseudohypertrophy, muscle atrophy, exercise-induced myalgia, macroglossia, and dysphagia $[7,8,10]$. There are three main clinical presentation patterns: 1) skeletal pseudohypertrophy with macroglossia and palpable
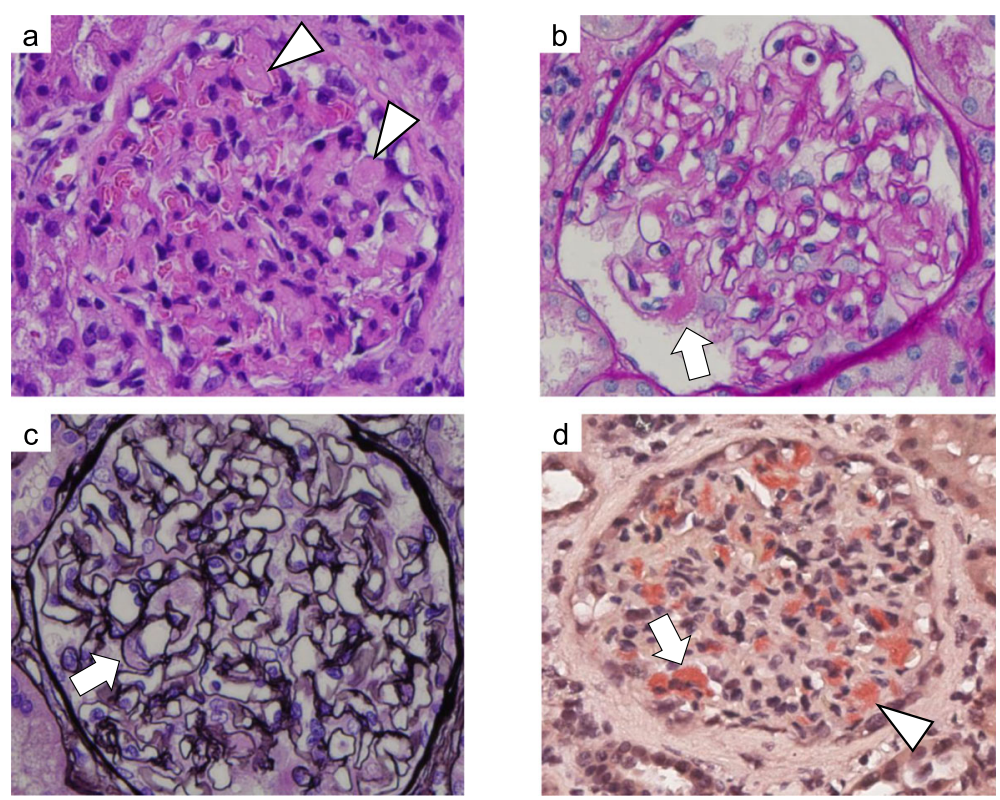

Fig. 4 Renal biopsy specimen on light microscopy. Hematoxylin and eosin staining $(\mathbf{a} ; \times 400)$, periodic acid-Schiff staining $(\mathbf{b} ; \times 400)$, and periodic acid-methenamine-silver staining $(\mathbf{c} ; \times 400)$ in light microscopy show an accumulation of eosinophilic and amorphous deposits in the mesangial region (white arrowheads) and part of the capillary walls (white arrows). Direct fast scarlet staining (Panel $d ; \times 400)$ shows amyloid proteins in the mesangial region (white arrowhead) and part of the capillary walls (white arrow) 

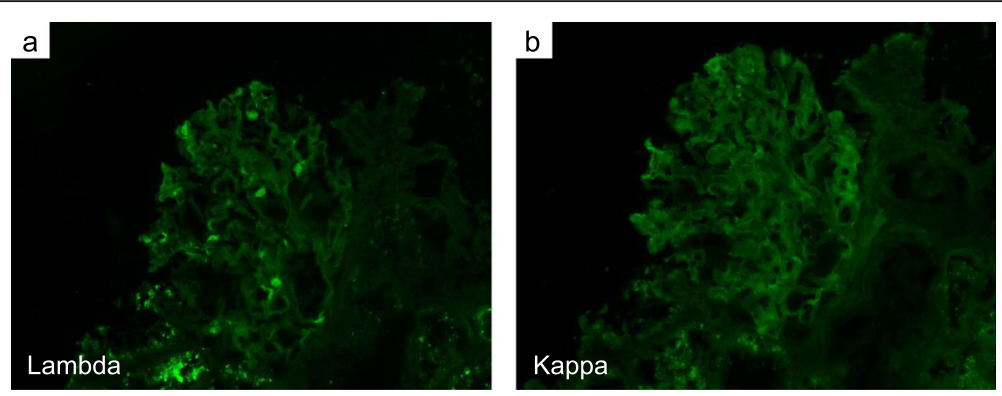

Fig. 5 Renal biopsy specimen on immunofluorescence microscopy. Direct immunofluorescence microscopy shows (1+) staining for lambda light chains $(\mathbf{a} ; \times 200)$ in the mesangial region and negative staining for kappa light chains $(\mathbf{b} ; \times 200)$

nodules in the muscles; 2) muscle weakness and atrophy only without other signs such as pseudohypertrophy or macroglossia; and 3) a combination of these two forms [7-10]. This elderly male patient presented with predominantly proximal symmetrical muscle weakness and muscle atrophy, which are common in amyloid myopathy, and muscle weakness of the neck and fingers, which is not common in amyloid myopathy. He had the second type of clinical presentation pattern, which has been reported to be particularly difficult to diagnose because the symptoms are nonspecific and may mimic those of polymyositis and primary inflammatory rheumatic diseases $[7-9,13,14]$. The electrophysiologic and pathological findings are also nonspecific, except for amyloid positivity [7-9, 14]. Because of its rarity and frequent misdiagnosis due to nonspecific presentations, a delayed diagnosis of the complication of amyloid myopathy is common (median time from first symptom to diagnosis is $17.5-23$ months) $[7,9,10]$. Hematologic response is an essential prerequisite for maintaining or improving organ functions and prolonging survival. A delay in chemotherapy initiation (and consequently in hematologic response) results in further deposition of amyloid that reduces the chances of improvement of organs, suggesting that early diagnosis and therapeutic intervention are important $[1,7,9,15]$. In order to diagnose amyloid myopathy without delay, confirming the presence of monoclonal protein in the blood or urine and amyloid positivity in muscle biopsy is important [8]. The percentage of multiple myeloma in $\mathrm{AL}$ amyloidosis was only $15 \%$ and even MGUS could cause AL amyloidosis [4]. In this case, the time from onset of muscle weakness to diagnosis of amyloid myopathy was only 10 months because we suspected the complication of amyloid myopathy due to the presence of MGUS (BJPlambda) and AL amyloidosis detected by renal biopsy. We diagnosed this patient with amyloid myopathy based on the Congo red positivity with birefringence in muscle biopsy (electron microscopic study was not available). Although lambda light chains were positive in renal biopsy, they were negative in muscle biopsy. We believe that this may be related to technical problems and limitations in light chain staining in muscle biopsy. Only a few reports have performed light chain staining in muscle biopsy $[8,14,16,17]$, and further research is thus necessary. Although we considered fine cytoplasmic inclusions, which were observed in one atrophic fiber on modified Gomori trichrome stain in muscle biopsy, were reminiscent of nemaline bodies, we could not confirm the nature of this structure and did not diagnose the
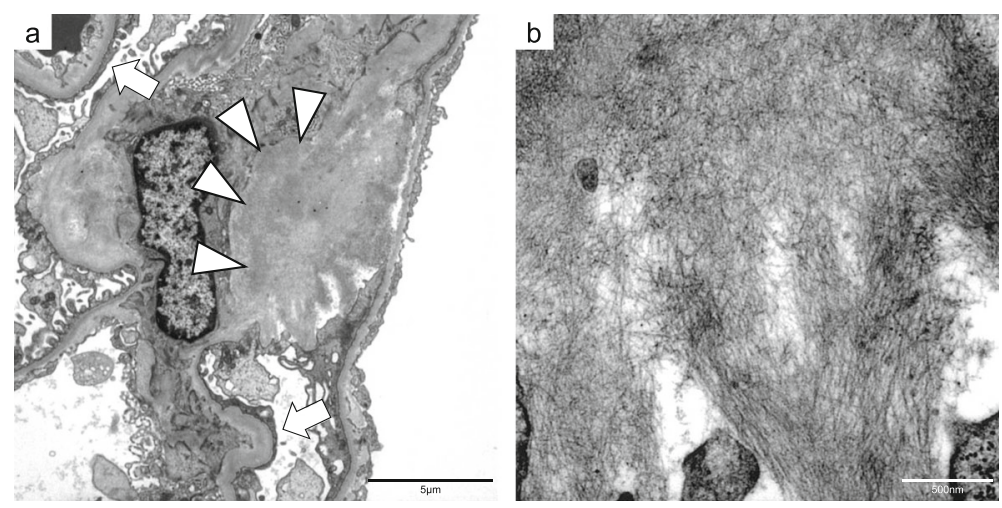

Fig. 6 Renal biopsy specimen on electron microscopy. Electron microscopy $(\mathbf{a} ; \times 4000, \mathbf{b} ; \times 30,000)$ shows amyloid fibrils in the mesangial region (white arrowheads) and podocyte foot process effacement (white arrows). The fiber diameter is between $9 \mathrm{~nm}$ and $12 \mathrm{~nm}$ 
patient with SLONM. However, if patients with MGRS including $\mathrm{AL}$ amyloidosis present with muscle weakness of the neck and fingers, we consider it important to include SLONM in the differential diagnoses. The reasons are as follows: 1) this symptom is reported to be relatively rare in amyloid myopathy $[7,8,10]$ but common in SLONM [18-21]; 2) half of SLONM patients were reported to have MGUS [12, 18]; and 3) the prognosis of SLONM-MGUS is poor, but certain treatments were reported to be effective in some cases [12, 18, 21-23].

The standard first-line treatment of AL amyloidosis is high-dose melphalan followed by autologous peripheral blood stem cell transplantation (HDM-SCT) [5]. For those patients who are ineligible for transplantation, second-line treatment such as cyclophosphamide, bortezomib, and dexamethasone (CyBorD) or third-line treatment such as melphalan and dexamethasone (MD) are recommended [5]. In this case, the patient was ineligible for HDM-SCT because of age, performance status, serum troponin $\mathrm{T}$ level, and a history of multiple organ failures of the kidneys, muscles, and heart. Furthermore, CyBorD, which has a side effect of cardiotoxicity, was also ineligible because the patient had a possible cardiac amyloidosis complication. We thus started MD treatment but neither the muscle weakness nor nephrotic syndrome improved. Although it was reported that MD was the most common treatment in amyloid myopathy and improved survival in patients whose survival was less than 8 years, only 8 of 23 patients with amyloid myopathy responded to treatment, including other therapy [7, 8, 24]. Daratumumab, pomalidomide, and doxycycline are promising agents for refractory AL amyloidosis $[5,24]$. We are also considering adding another therapy in the future.

The prognostic factor of AL amyloidosis is the complication of cardiac involvement, and the median survival duration was reported to be 55 months in Revised Mayo stage 1, 19 months in stage 2, 12 months in stage 3, and 5 months in stage 4 . This case was classified as Revised Mayo stage 3 based on the serum troponin $\mathrm{T}$ level $(\geq 0.025 \mathrm{ng} / \mathrm{mL})$ and the difference between the involved and uninvolved serum free light chain $(\geq 180 \mathrm{mg} / \mathrm{L})$ [6]. The prognosis of renal amyloidosis or amyloid myopathy is also poor (approximately 22-32 months from onset of symptoms due to amyloid myopathy to death) $[7,8,25]$. This patient lived for at least 21 months despite the fact that he had no improvement in symptoms. In contrast, the prognostic factor of SLONM is the complication of MGUS, and the majority of SLONM-MGUS patients are reported to die from respiratory failure within a few years [12]. In the future, in cases in which the complication of SLONM is suspected, we should carefully monitor the patients for both cardiac dysfunction due to the progression of cardiac amyloidosis and respiratory failure due to the progression of respiratory muscle atrophy.

In summary, we report a rare case of MGRS-related $\mathrm{AL}$ amyloidosis complicated by amyloid myopathy. AL amyloidosis involving the kidneys and muscles has a poor prognosis, and a delayed diagnosis of amyloid myopathy is common because of its rarity and frequent misdiagnosis, which increases organ function deterioration. Therefore, early detection, therapeutic intervention, and careful follow-up are crucial.

\begin{abstract}
Abbreviations
AChR: Acetylcholine receptor; AL: Amyloid immunoglobulin light chain; AMA2: Anti-mitochondrial M2; ANA: Antinuclear antibodies; anti-ARS: Antiaminoacyl tRNA synthetase; anti-CCP: Anti-cyclic citrullinated peptide; antidsDNA: Anti-double-stranded DNA; anti-MuSK: Anti-muscle-specific kinase; BNP: Brain natriuretic peptide; BJP: Bence Jones protein; C3: Complement component 3; CyBorD: Cyclophosphamide, bortezomib, and dexamethasone; HDM-SCT: High-dose melphalan followed by autologous peripheral blood stem cell transplantation; HE: Hematoxylin and eosin; Ig: Immunoglobulin; MD: Melphalan and dexamethasone; MGRS: Monoclonal gammopathy of renal significance; MGUS: Monoclonal gammopathy of undetermined significance; MMT: Manual muscle testing; M protein: Monoclonal protein; MRI: Magnetic resonance imaging; NADH-TR: Nicotinamide adenine dinucleotide-tetrazolium reductase; PGNMID: Proliferative glomerulonephritis with monoclonal Ig deposits; SLONM: Sporadic late-onset nemaline myopathy; SLONM-MGUS: SLONM with MGUS; STIR: Short inversion time inversion recovery
\end{abstract}

\section{Acknowledgements}

Not applicable.

\section{Authors' contributions}

$\mathrm{EO}, \mathrm{Al}, \mathrm{YH}, \mathrm{NK} 1, \mathrm{TA}, \mathrm{KT}$, and $\mathrm{ST}$ contributed to the data collection; EO and $\mathrm{Al}$ designed the research. EO, Al, NK1, TA, NK2, KS, SE, HY, TM, SM, IN, and MY evaluated the pathological tissue. EO performed primary manuscript preparation; $\mathrm{EO}$ and $\mathrm{Al}$ wrote the paper. Al had primary responsibility for the final content; and EO, Al, NK1, TA, NK2, KS, SE, HY, TM, SM, IN, RT, and MY reviewed the paper and revised it critically. All authors read and approved the final manuscript.

\section{Funding}

This study was partly supported by an Intramural Research Grant $(2-5,29-4)$ for Neurological and Psychiatric Disorders of the National Center of Neurology and Psychiatry. The funders had no role in study design, data collection, statistical analysis, decision to publish, or preparation of the manuscript.

\section{Availability of data and materials}

The dataset supporting the conclusions of this article is included within the article.

Ethics approval and consent to participate

This case report was written in compliance with the Declaration of Helsinki.

\section{Consent for publication}

Written consent for publication of this case report was obtained from the patient.

\section{Competing interests}

The authors declare that they have no competing interests.

\section{Author details}

'Department of Nephrology, Graduate School of Medicine, Kyoto University, 54 Shogoin Kawahara-cho, Sakyo-ku, Kyoto 606-8507, Japan. ${ }^{2}$ Department of Neurology, Graduate School of Medicine, Kyoto University, 54 Shogoin-Kawahara-cho, Sakyo-ku, Kyoto 606-8507, Japan. ${ }^{3}$ Department of Diagnostic Pathology, Graduate School of Medicine, Kyoto University, 54 
Shogoin-Kawahara-cho, Sakyo-ku, Kyoto 606-8507, Japan. ${ }^{4}$ Department of Neuromuscular Research, National Institute of Neuroscience, National Center of Neurology and Psychiatry (NCNP), 4-1-1 Ogawa-Higashi, Kodaira, Tokyo 187-8502, Japan.

Received: 30 May 2020 Accepted: 18 February 2021

Published online: 27 February 2021

\section{References}

1. Bridoux F, Leung N, Hutchison CA, Touchard G, Sethi S, Fermand JP, et al. Diagnosis of monoclonal gammopathy of renal significance. Kidney Int. 2015;87:698-711.

2. Leung N, Bridoux F, Batuman V, Chaidos A, Cockwell P, D'Agati VD, et al. The evaluation of monoclonal gammopathy of renal significance: a consensus report of the international kidney and monoclonal Gammopathy research group. Nat Rev Nephrol. 2019;15:45-59.

3. Rosner MH, Edeani A, Yanagita M, Glezerman IG, Leung N, American Society of Nephrology Onco-Nephrology Forum. Paraprotein-related kidney disease: diagnosing and treating monoclonal gammopathy of renal significance. Clin J Am Soc Nephrol. 2016;11:2280-7.

4. Leung N, Bridoux F, Hutchison CA, Nasr SH, Cockwell P, Fermand JP, et al. Monoclonal gammopathy of renal significance: when MGUS is no longer undetermined or insignificant. Blood. 2012;120:4292-5.

5. Milani P, Merlini G, Palladini G. Light chain amyloidosis. Mediterr J Hematol Infect Dis. 2018;10:e2018022.

6. Kumar S, Dispenzieri A, Lacy MQ, Hayman SR, Buadi FK, Colby C, et al. Revised prognostic staging system for light chain amyloidosis incorporating cardiac biomarkers and serum free light chain measurements. J Clin Oncol. 2012;30:989-95.

7. Muchtar E, Derudas D, Mauermann M, Liewluck T, Dispenzieri A, Kumar SK, et al. Systemic immunoglobulin light chain amyloidosis-associated myopathy: presentation, diagnostic pitfalls, and outcome. Mayo Clin Proc. 2016:91:1354-61

8. Chapin JE, Kornfeld M, Harris A. Amyloid myopathy: characteristic features of a still underdiagnosed disease. Muscle Nerve. 2005;31:266-72.

9. Accardi F, Papa V, Capozzi AR, Capello GL, Verga L, Mancini C, et al. A rare case of systemic $\mathrm{AL}$ amyloidosis with muscle involvement: a misleading diagnosis. Case Rep Hematol. 2018;2018:9840405.

10. Liewluck T, Milone M. Characterization of isolated amyloid myopathy. Eur J Neurol. 2017;24:1437-45.

11. Engel AG. Late-onset rod myopathy (a new syndrome?): light and electron microscopic observations in two cases. Mayo Clin Proc. 1966:41:713-41.

12. Chahin N, Selcen D, Engel AG. Sporadic late onset nemaline myopathy. Neurology. 2005:65:1158-64.

13. Perfetto F, Moggi-Pignone A, Livi R, Tempestini A, Bergesio F, MatucciCerinic M. Systemic amyloidosis: a challenge for the rheumatologist. Nat Rev Rheumatol. 2010;6:417-29.

14. Mandl LA, Folkerth RD, Pick MA, Weinblatt ME, Gravallese EM. Amyloid myopathy masquerading as polymyositis. J Rheumatol. 2000;27:949-52.

15. Merlini G, Stone MJ. Dangerous small B-cell clones. Blood. 2006;108:2520-30.

16. Spuler S, Emslie-Smith A, Engel AG. Amyloid myopathy: an underdiagnosed entity. Ann Neurol. 1998:43:719-28.

17. Ostrow LW, Corse AM, Morrison BM, Huff CA, Carrino JA, Hoke A, et al. Expanding the spectrum of monoclonal light chain deposition disease in muscle. Muscle Nerve. 2012;45:755-61.

18. Schnitzler L, Schreckenbach T, Nadaj-Pakleza A, Stenzel W, Rushing EJ, Van Damme $P$, et al. Sporadic late-onset nemaline myopathy: clinic-pathological characteristics and review of 76 cases. Orphanet J Rare Dis. 2017;12:86.

19. Uruha A, Benveniste O. Sporadic late-onset nemaline myopathy with monoclonal gammopathy of undetermined significance. Curr Opin Neurol. 2017:30:457-63.

20. Monforte M, Primiano G, Silvestri G, Mirabella M, Luigetti M, Cuccagna C, et al. Sporadic late-onset nemaline myopathy: clinical, pathology and imaging findings in a single center cohort. J Neurol. 2018;265:542-51.

21. Nakamura K, Shibuya K, Nishino I, Kuwabara S. Dropped head in sporadic late-onset nemaline myopathy. Intern Med. 2019;58:1967-8.

22. Mizuno Y, Mori-Yoshimura M, Okamoto T, Oya Y, Nishino I, Murata M. [Two cases of sporadic late onset nemaline myopathy effectively treated with immunotherapy]. Rinsho Shinkeigaku. 2016; 56:605-11. Japanese.

23. Kumutpongpanich T, Owattanapanich W, Tanboon J, Nishino I, Boonyapisit K. Sporadic late-onset nemaline myopathy with monoclonal gammopathy of undetermined significance (SLONM-MGUS): an alternative treatment using cyclophosphamide-thalidomide-dexamethasone (CTD) regimen. Neuromuscul Disord. 2018;28:610-3

24. Gertz MA. Immunoglobulin light chain amyloidosis diagnosis and treatment algorithm 2018. Blood Cancer J. 2018;8:44.

25. Kuroda T, Tanabe N, Kobayashi D, Wada Y, Murakami S, Nakano M, et al. Significant association between renal function and amyloid-positive area in renal biopsy specimens in AL amyloidosis. BMC Nephrol. 2012;13:118.

\section{Publisher's Note}

Springer Nature remains neutral with regard to jurisdictional claims in published maps and institutional affiliations.
Ready to submit your research? Choose BMC and benefit from:

- fast, convenient online submission

- thorough peer review by experienced researchers in your field

- rapid publication on acceptance

- support for research data, including large and complex data types

- gold Open Access which fosters wider collaboration and increased citations

- maximum visibility for your research: over $100 \mathrm{M}$ website views per year

At $\mathrm{BMC}$, research is always in progress.

Learn more biomedcentral.com/submissions 